\title{
Many-body localization from random magnetic anisotropy
}

\author{
Jie Gu ${ }^{\circ}$, Shuanglong Liu, Maher Yazback, Hai-Ping Cheng, and X.-G. Zhang* \\ Department of Physics, Center for Molecular Magnetic Quantum Materials and Quantum Theory Project, \\ University of Florida, Gainesville, Florida 32611, USA
}

(Received 10 October 2019; revised manuscript received 19 November 2019; published 18 December 2019)

\begin{abstract}
One of the main difficulties of observing many-body localization in natural solid-state materials is creating strong enough disorder. A strong random local magnetic field is difficult to achieve in a solid state material. We propose exploiting large random magnetic anisotropy, either in magnitude or direction, which can be realized in organometallic quantum magnets. We present the phase diagram of an $S=1$ Heisenberg chain in terms of both a random magnetic anisotropy and a random magnetic field. The many-body localization phase emerges with sufficiently large anisotropy under very small random fields. We propose candidate materials of doped single-chain organometallic quantum magnets for realizing many-body localization, where either orientation disorder or substitution of metal ions can create large random magnetic anisotropy required in our prediction.
\end{abstract}

DOI: 10.1103/PhysRevResearch.1.033183

\section{INTRODUCTION}

Recently, extensive theoretical [1-3], numerical [4-7], and experimental [8-14] evidence establishes that Anderson localization [15], the suppression of transport due to quenched disorder, can survive interactions for sufficiently strong disorder. Such localization in the presence of interactions is now known as many-body localization (MBL) [16-19] and is understood as an exception of the eigenstate thermalization hypothesis (ETH) [20,21], the foundation for understanding the emergence of statistical mechanics in quantum mechanical systems under unitary dynamics. Such localization effects can impact not only electronics but also phononics [22], photonics $[23,24]$, and presumably magnonics [25]. MBL enables slow dephasing [26], long-term memory of local initial states, and localization-protected order [27], all of which may offer great potential for applications in quantum computing.

Many-body localization has been experimentally observed in fine-tuned artificial experimental platforms, including ultracold atoms [8-10,13], trapped ions [11], a dipolar spin system in diamond [12], and recently nuclear spins in a solid state system [14], but it is yet to be observed in natural electronic systems. Solid state spin systems can provide one possible platform for experimental observation. Such spin systems were considered in Anderson's original paper. They are also popular in the studies of MBL due to both their simplicity [5-7] and experimental accessibility [11,14]. Disorder due to a random magnetic field is used in most theoretical studies $[5,11]$.

\footnotetext{
*xgz@ufl.edu
}

Published by the American Physical Society under the terms of the Creative Commons Attribution 4.0 International license. Further distribution of this work must maintain attribution to the author(s) and the published article's title, journal citation, and DOI.
Instead of random field, random magnetic anisotropy is more commonly seen in amorphous magnets [28]. We can describe such materials using the following Hamiltonian (in 1D):

$$
\mathcal{H}=\sum_{i}^{L}\left[J \mathbf{S}_{i} \cdot \mathbf{S}_{i+1}+h_{i} S_{i}^{z}-D_{i}\left(\mathbf{n}_{i} \cdot \mathbf{S}\right)^{2}\right],
$$

where $J$ is the exchange coupling, $h_{i}, D_{i}$, and $\mathbf{n}_{i}$ are magnetic fields, magnetic anisotropy, and the anisotropy axis at each site $i$, respectively. There have been few studies of localization phenomena for spin waves in magnets with random anisotropy [29-33], and these mostly used a semiclassical approach and focused on the low-energy excitations, neglecting magnonmagnon interaction. On the other hand, in the context of MBL, even though no studies on random-anisotropy spin chains have been reported, MBL has been studied in a closely related model, the Bose-Hubbard model. The Hamiltonian (1) can be transformed to the Bose-Hubbard model (plus higher-order terms) by using the Holstein-Primakoff transformation $S_{i}^{-}=$

$$
\begin{aligned}
& \sqrt{2 S-n_{i}} b_{i}, S_{i}^{+}=\left(S_{i}^{-}\right)^{\dagger}, S_{i}^{z}=n_{i}-S, \\
& \mathcal{H}=-t \sum_{\langle i, j\rangle}\left(b_{i}^{\dagger} b_{j}+b_{j}^{\dagger} b_{i}\right)+\frac{U}{2} \sum_{i} n_{i}\left(n_{i}-1\right)-\mu \sum_{i} n_{i} \\
& \quad+\text { higher-order terms, }
\end{aligned}
$$

where $t=-J S, \quad U=-2 D_{i} \cos ^{2} \theta_{i}$, and $\mu=2 J S-h_{i}+$ $D_{i}\left[S-(3 S-1) \cos ^{2} \theta_{i}\right]$. Previous works have given numerical evidence for the MBL phase in the Bose-Hubbard model a random on-site potential [34] or random on-site interaction [35]. A spin chain with random anisotropy is equivalent to a Bose-Hubbard system, with high-order terms added, where $U$ and $\mu$ are random but correlated.

Here we numerically study the many-body localization transition (MBLT) in the $S=1$ spin chain using the Heisenberg model with random magnetic anisotropies and under a locally random magnetic field. The central result is a random field strength $W_{h}$-anisotropy $D$ phase diagram. Based on this 
phase diagram we discuss the possible realization of MBL in solid state electronic materials, particularly, in disordered organometallic quantum magnets.

\section{MANY-BODY LOCALIZATION TRANSITION BY RANDOM ANISOTROPY}

Due to the absence of magnetic anisotropy in $S=1 / 2$ system, $S=1$ chain is the simplest system with magnetic anisotropy and the most suitable for numerical study using exact diagonalization. The Hamiltonian in Eq. (1) describes an $S=1$ chain of length $L$ with random fields and random anisotropy, where the random fields $h_{i}$ at each site are drawn from a uniform distribution $\left[-W_{h}, W_{h}\right]$. The anisotropy constants $D_{i}$ can be either uniform $D_{i}=D$ or drawn from $\left[0, W_{D}\right]$ for $W_{D}>0$ or $\left[W_{D}, 0\right]$ for $W_{D}<0$. The anisotropy axis $\mathbf{n}_{i}$ can also be either uniform (along $z$ axis) or drawn from the unit sphere. Periodic boundary conditions are used. In the limit of $J=0$, the many-body eigenstates are simple product states and the system is trivially localized. Therefore we consider a finite $J$, which is set as 1 hereafter.

By increasing the strength of randomness of the parameters above, we anticipate a transition from the thermal phase to the MBL phase. Many properties can be used to distinguish the two phases. Here we use two popular criteria: the mean difference in local observables between adjacent eigenstates [5] and spectral statistics [4]. Each of the criteria is first averaged over more than 50 eigenpairs in the middle of the spectrum for each sample and then averaged over samples (disorder realizations). For the finite size $L=8,10$, and 12 considered in this work, we calculated 3000,300 , and 300 samples, respectively. Consider the expectation value of $\left(S^{z}\right)^{2}$ at site $i$ for the eigenstate $n$ of sample $\alpha$

$$
m_{i \alpha}^{(n)}=\left\langle n\left|\left(S_{i}^{z}\right)^{2}\right| n\right\rangle .
$$

ETH requires that the difference in $m_{i \alpha}^{(n)}$ between adjacent eigenstates vanishes exponentially with increasing system size. Thus this difference can serve as an indicator for MBL. The logarithm of averaged $\left|m_{i \alpha}^{(n)}-m_{i \alpha}^{(n+1)}\right|, \bar{m}$, as a function of system size $L$ can be fitted by a linear function

$$
\ln \bar{m}=\mu L+b .
$$

In the ergodic (thermal) phase, $\mu$ is negative and finite, while in the MBL phase $\mu$ is zero.

Another diagnostic we use is the spectral statistics. In the thermal phase, the energy spectrum has level statistics of Gaussian-orthogonal ensemble (GOE) while a Poissonian (or paired-Poisson) level statistics is expected for the localized phase. These two types of statistics are manifested in the ratio of adjacent energy gaps

$$
r_{n}=\min \left(\delta_{n}, \delta_{n-1}\right) / \max \left(\delta_{n}, \delta_{n-1}\right),
$$

where $\delta_{n}=E_{n}-E_{n-1}$ and $E_{n}$ 's are ordered many-body eigenenergies. The average, $r$, of $r_{n}$ over states changes from $r_{\mathrm{GOE}} \simeq 0.53$ for the thermal phase [36] to $r_{\text {Poisson }}=2 \ln 2-$ $1 \simeq 0.39$ for the MBL phase [4].

To simplify the calculation, we consider only states with $S_{\text {total }}^{z}=0$, which have the largest density of states. The Hamiltonian with random anisotropy magnitude and fixed

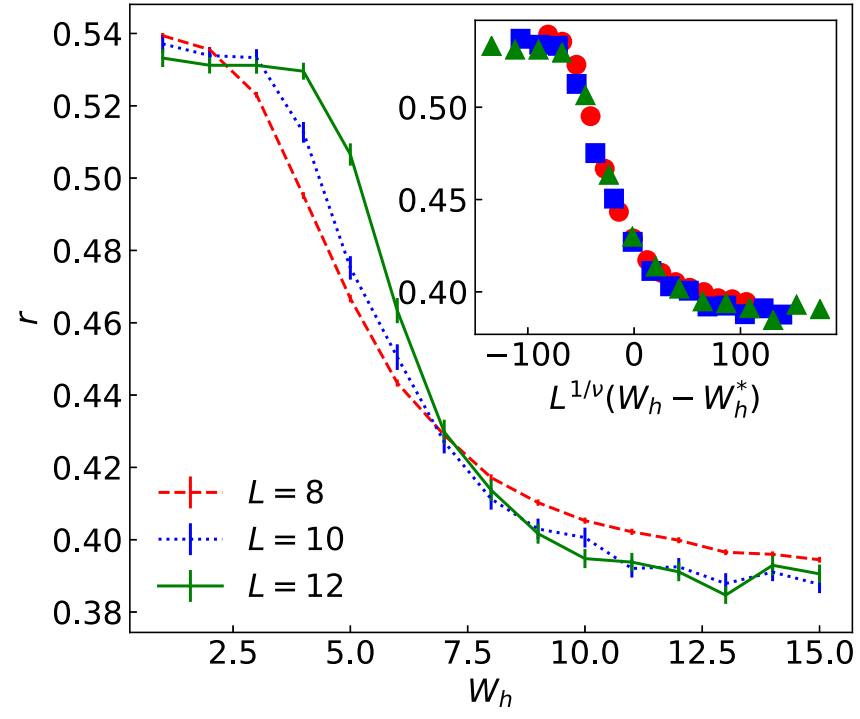

FIG. 1. Average ratios of adjacent energy gaps as a function of random field strength $W_{h}$ for eigenpairs in the middle of the spectrum. The inset shows that the curves for different system size $L$ are collapsed by a scaling form $L^{1 / v}\left(W_{h}-W_{h}^{*}\right)$, where $W_{h}^{*}=$ $7.09 \pm 0.19$ and $v=1.25 \pm 0.10$.

anisotropy axis conserves $S_{\text {total }}^{z}$, so nonzero $S_{\text {total }}^{z}$ states will not be mixed in by dynamics. But the Hamiltonian with random anisotropy axis does not conserve $S_{\text {total }}^{z}$. However, even in this case we can still assume that $S_{\text {total }}^{z}$ stays close to zero. Therefore, in the rest of the paper, we will focus on the $S_{\text {total }}^{z}=0$ sector only. The shift-invert method [37] will be used to calculate the eigenpairs in the middle of the energy spectrum, corresponding to the infinite temperature if the states are thermal.

To make contact with results in the literature $[5,7,38]$, we first consider the limit of zero anisotropy. The ratio of adjacent energy gaps $r$ as a function of the random field strength $W_{h}$ is shown in Fig. 1. As $W_{h}$ is increased, we can see a transition from the thermal phase to MBL phase at $W_{h}^{*} \sim 8$. A more exact value of the critical random field strength $W_{h}^{*}$ can be obtained via a finite-size scaling analysis. Scaling the disorder strength $W_{h}$ with a form $x=L^{1 / v}\left(W_{h}-W_{h}^{*}\right)$ yields a scaling collapse of all data points (see inset of Fig. 1). Values of $W_{h}^{*}$ and $v$ that give the best collapse are found to be $W_{h}^{*}=$ $7.09 \pm 0.19$ and $v=1.25 \pm 0.10$. The statistical averages and the error bars are calculated in the following manner. After obtaining $r$ for all the samples, at each $W_{h}$ we bootstrap from the original set of $r$ values to generate a new set containing the same number of $r$ values then find their average. These averages for all $W_{h}$ are used to find a pair of $W_{h}^{*}$ and $v$ that give the best collapse on a generalized logistic function in a least-squares sense. This process is repeated 3000 times to produce the average and standard deviation of those pairs.

The critical disorder $W_{h}^{*} \simeq 7.09$ for $S=1$ chain is significantly larger than that of $S=1 / 2, W_{h}^{*(S=1 / 2)} \simeq 3.7$, which is consistent among different studies [7,38]. This can be understood from observing that in the Hamiltonian (2), the field term $h_{i}$ in $\mu$ does not depend on $S$, but all other nonzero low-order terms scale with $S$. This observation leads to a 


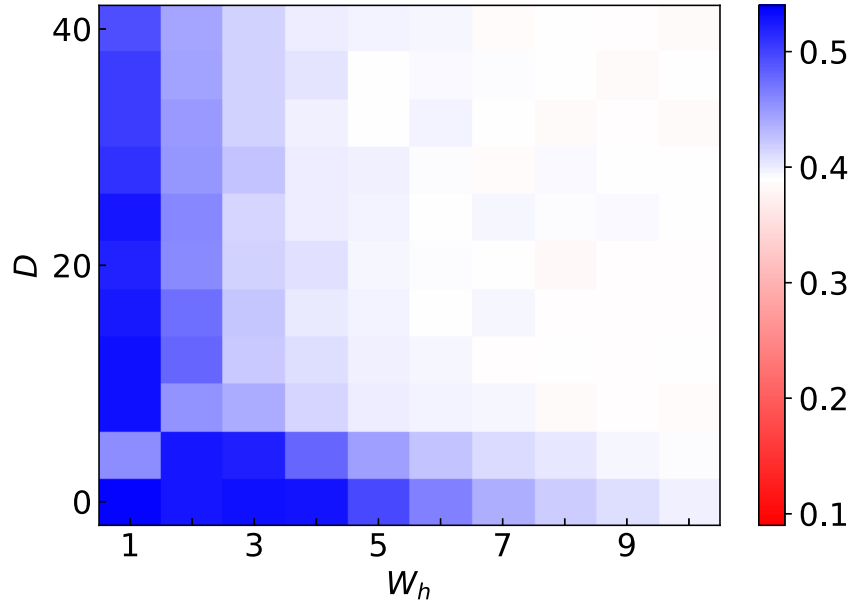

FIG. 2. Random field strength $W_{h}$-uniform anisotropy strength $D$ phase diagram. Colors represent $r$, the averaged ratios of adjacent energy gaps over the eigenstates in the middle of the spectrum. When $D$ increases, the critical random field $W_{h}^{*}$ decreases rapidly until approaching a limit value of about 4 .

scaling of the critical field parameter $W_{h}^{*(S=1)}=2 W_{h}^{*(S=1 / 2)} \simeq$ 7.4 , close to the actual value of 7.09.

Next we introduce the effect of a uniform magnetic anisotropy $D_{i}=D$ with fixed anisotropy axis. The effect of $D$ on MBLT is shown in Fig. 2. The energy spectrum in the limit of $J=0$ is independent of the overall sign of the Hamiltonian, except for the addition of an offset. This means that the eigenpairs in the middle of the spectrum have approximate symmetry between $D$ and $-D$ when $|D / J| \gg 1$, and therefore it is sufficient to show only positive $D$ in the figure. The critical random field $W_{h}^{*}$ decreases rapidly with increasing anisotropy $D$, but appears to approach a finite value of about 4 when $D \gtrsim 10$.

The more interesting case is the random anisotropy axis model. To simulate this model, we keep a constant $D$ across different sites but randomly select $\mathbf{n}_{i}$, the direction of the anisotropy axis, of each site from the unit sphere. Using the diagnostic $\mu$, the effect of $D$ on MBLT is shown in Fig. 3, the random field strength $W_{h}$-anisotropy $D$ phase diagram. The approximate symmetry between $D$ and $-D$ can be seen from the phase diagram. The critical random field $W_{h}^{*}$ decreases with the increasing random anisotropy $D$, but instead of saturating in Fig. 2, there is a critical random anisotropy $D^{*} \sim 10$ beyond which any small $W_{h}$ enables MBL. Our simulations show that random anisotropy magnitude with fixed anisotropy axis and random axis orientations with fixed magnitude give quantitatively similar results, i.e., replacing $D$ by $W_{D}$ in Fig. 3 gives an approximate phase diagram for random anisotropy magnitude with axis fixed, so here we present only the results of random axis. A natural question is whether there is MBLT at $W_{h}=0$. Figure 4 gives $r$ as a function of $W_{h}$ and $D$ for different system sizes, including the extreme case $W_{h}=0$. The phase boundaries in these subfigures are consistent with Fig. 3 except for extra phase boundaries between $r<0.39$ (red) and $r=0.39$ (white). Inside the MBL phase (upper left) this indicates a spectral transition from Poisson to pairedPoisson statistics, since (almost) every eigenstate comes in

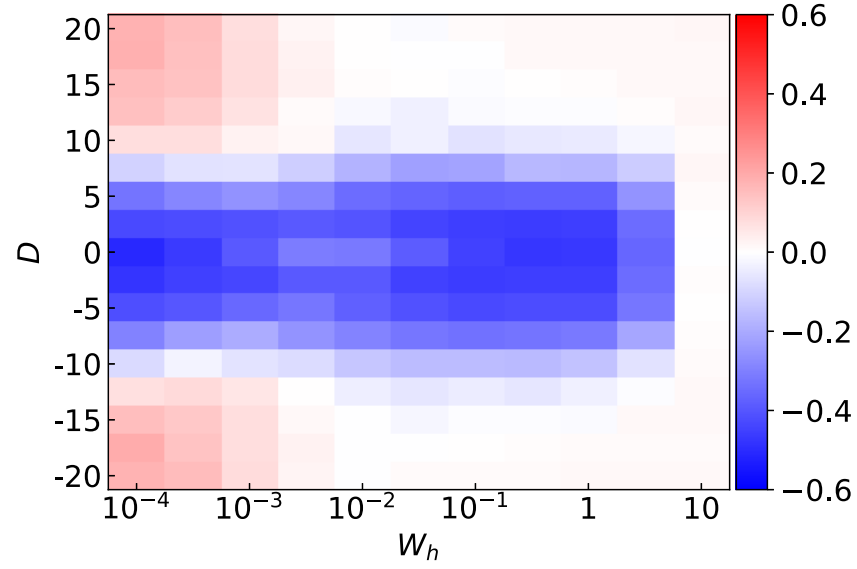

FIG. 3. Random field strength $W_{h}$-anisotropy $D$ phase diagram. The colors represent $\mu$ defined in Eq. (3), characterizing how the spin expectation value fluctuates between adjacent eigenstates. $\mu=0$ indicates the violation of ETH, i.e., MBL phase, and $\mu<0$ is the ergodic phase.

pairs and $r=0$ in the limit of infinite $D$. The paired-Poisson regime shrinks with the increasing system size as shown in Fig. 4. This is because larger systems have smaller gaps $\left(\propto D L / 3^{L}\right)$, so the energy splitting by exchange coupling and fields has a larger impact on the spectrum. Therefore it is more difficult for large systems to enter the paired-Poisson regime, and this spectral transition is merely a size effect and absent in the thermodynamic limit. The $D \rightarrow \infty, W_{h}=0$ point becomes singular in the sense that $r=0$ at that point while elsewhere in the MBL phase $r=0.39$.

\section{POSSIBLE REALIZATION OF MBL IN ORGANOMETALLIC QUANTUM MAGNETS}

In this section, we discuss the feasibility of realizing MBL in naturally disordered materials, particularly in organometallic quantum magnets. There is a large variety of magnetic materials including the more traditional inorganic ones and the newer organometallic hybrid materials, e.g., single molecule magnets (SMMs) [39], single chain magnets (SCMs) [40], magnetic metal-organic frameworks (MOFs) [41] etc. One of the advantages of molecule-based metal-organic hybrid materials over traditional materials is their high customizability attributed to the great number of candidate organic ligands. The crystal field by the ligands around the metal ions is the source of the strong anisotropy in organometallic hybrid materials, and is sensitive to what ligand it is and how the ligand is connected with the core. Therefore substitution of either ligands [42] or metal ions [43] can be used to create disordered anisotropy magnitude. The exchange couplings between magnetic molecules are also bridged by the ligands, so the exchange couplings can be tuned by substituting ligands and be random as well [42]. The intermolecular interactions are weak, so it is feasible to rotate each single molecule and thus the magnetic anistropy can have different axes for each molecule. Moreover, molecular magnets are known for being well isolated from the environment and having long coherence times $(\sim 100 \mu \mathrm{s})$ [44-49]. Organometallic quantum magnets can be in $1 \mathrm{D}[40,50], 2 \mathrm{D}[51,52]$, and 3D [39,53], providing 
(a)

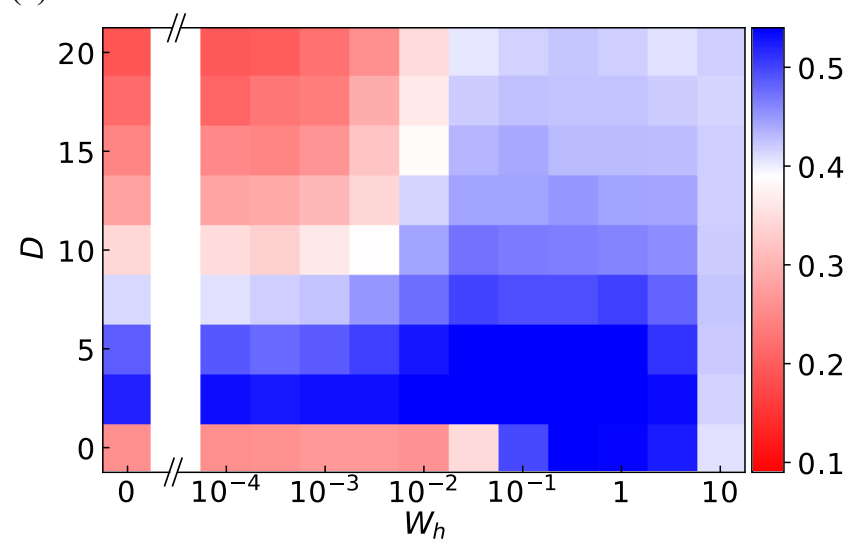

(b)

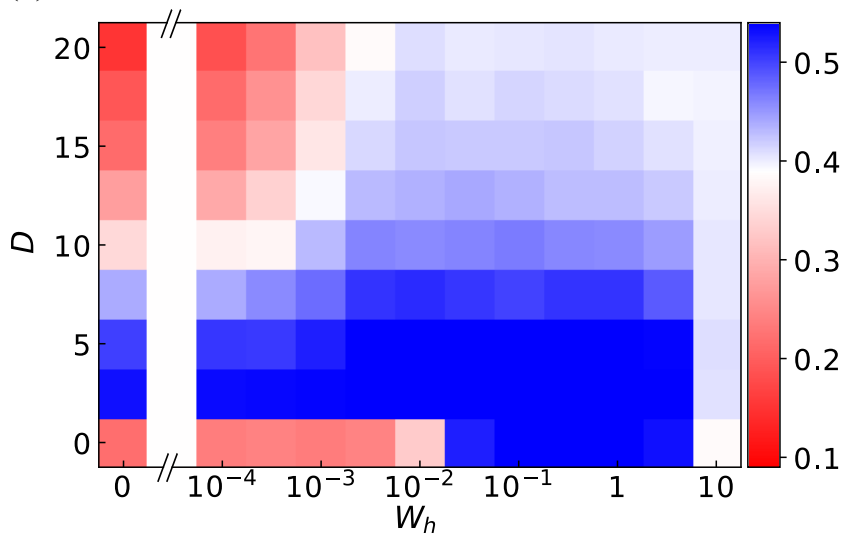

(c)

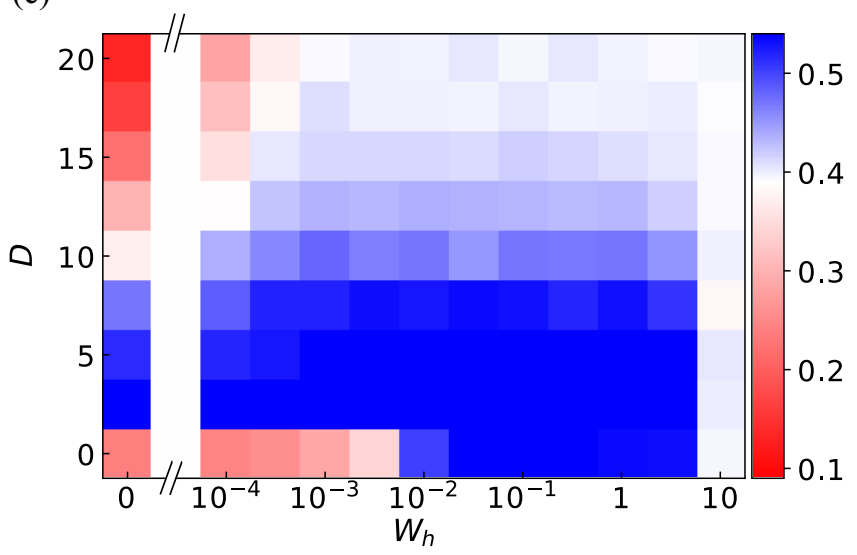

FIG. 4. Random field strength $W_{h}$-magnetic anisotropy $D$ phase diagram for system size (a) $L=8$, (b) 10 , and (c) 12. Colors represent $r$.

convenient experimental platforms for studying MBL beyond $1 \mathrm{D}$.

As shown in the phase diagram (Fig. 3), we expect a fully many-body localized phase in the $S=1$ Heisenberg chain with a uniform $|D / J| \gtrsim 10$ but random anisotropy axis. Spin-1 magnetic chains have been extensively studied both theoretically and experimentally. The well-known Haldane phase is expected in the range $-0.25 \leqslant D /|J|<0.97$ and observed in several (mostly inorganic) materials, e.g.,
TABLE I. Magnetic anisotropy constant $D$ of pure DTN, Br-doped DTN, and Idoped DTN from DFT calculations. The spins are treated quantum mechanically using the method in Ref. [58].

\begin{tabular}{ll}
\hline \hline & $D(\mathrm{~K})$ \\
\hline DTN & 6.3 \\
Br-DTN & 5.1 \\
I-DTN & 3.5 \\
\hline \hline
\end{tabular}

$\mathrm{CsNiCl}_{3}$ [54]. The single-ion anisotropy in inorganic materials is usually small $(<0.1 \mathrm{~K})$ and $D / J$ ratios much smaller than $1(<0.1)$, far below the required MBLT critical disorder. Organometallic materials are better candidates due to their large anisotropy and versatility of ligands, and have been used to experimentally study the "large- $D$ " phase [55]. We identify two most promising candidates among these materials. The first material is $\mathrm{Ni}\left(\mathrm{C}_{2} \mathrm{H}_{8} \mathrm{~N}_{2}\right)_{2} \mathrm{Ni}(\mathrm{CN})_{4}$, also known as NENC. Its $|D / J|$ ratio is reported to be $7.5 \sim 9.5[56,57]$ and a theoretical work, which provides a better and more consistent fit to the experimental data, shows that $|D / J|=$ $37.6(D=-6.4 \mathrm{~K}, J=0.17 \mathrm{~K})$ [55]. The second material, $\mathrm{Ni}\left(\mathrm{C}_{10} \mathrm{H}_{8} \mathrm{~N}_{2}\right)_{2} \mathrm{Ni}(\mathrm{CN})_{4} \cdot \mathrm{H}_{2} \mathrm{O}$ or $\mathrm{NBYC}$, has $|D / J|=12.7$ [57], even though the previous theoretical work gives 7.5 [55].

Another popular $S=1$ spin chain is $\mathrm{NiCl}_{2} \cdot 4 \mathrm{SC}\left(\mathrm{NH}_{2}\right)_{2}$ (DTN). Br-doped DTN is observed to exhibit Bose glass and Mott glass phases and has $D / J=4.0$ [42]. This is somewhat below our estimated threshold for MBLT. Both the experiment and density functional theory (DFT) calculations show a decrease in $D$ when $\mathrm{Cl}$ is substituted by $\mathrm{Br}$ or I (Table I). The seemingly counterintuitive trend of $D$ can be explained by the fact that the substituted atom is on the ligand not the core. DTN has an easy plane energies for both the out-of-easy-plane orientation and the in-easy-plane orientation are enhanced by doping heavier elements, but the enhancement for the in-plane orientation is stronger. To increase the anisotropy energy, a different doping strategy may be pursued.

We also studied the possibility of using external electric fields to enhance the magnetic anisotropy energy (MAE) of a DTN slab using DFT calculations, considering the strong magnetoelectric coupling in DTN [59]. The DTN slab is three unit cells thick, and the atomic structure is kept to be bulklike under both zero and finite out-of-plane electric fields. The atomic structure is fixed to better represent the bulk environment and surface relaxations are ignored. Both the magnetic hard axis and the bulk polarization are in-plane along the $\mathrm{Ni}-\mathrm{Cl}$ bonds, and the magnetic easy axis (along $\mathrm{Ni}-\mathrm{S}$ bonds) is perpendicular to the slab. When the electric field is smaller than $0.15 \mathrm{~V} / \AA$, the MAE is almost unchanged. As the electric field is increased beyond $0.15 \mathrm{~V} / \AA$, the MAE decreases rapidly (Fig. 5). Even though magnetic anisotropy was not enhanced in this calculation, it provides evidence for possible random anisotropy magnitudes tunable by a random electric field. Other directions of electric fields will be examined in a future study.

The required random orientation of molecules may be obtained in the following manner. Many magnetic molecules 


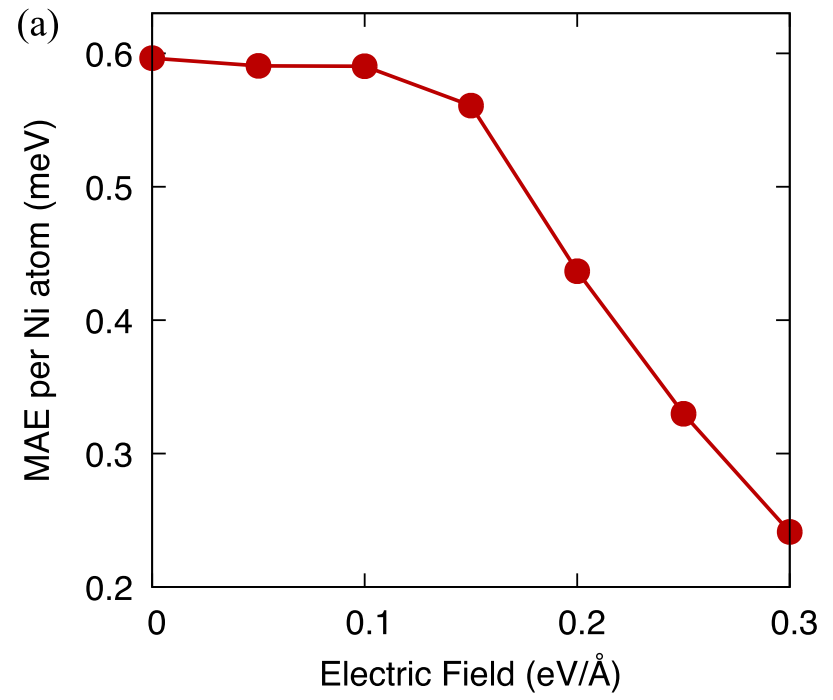

(b)

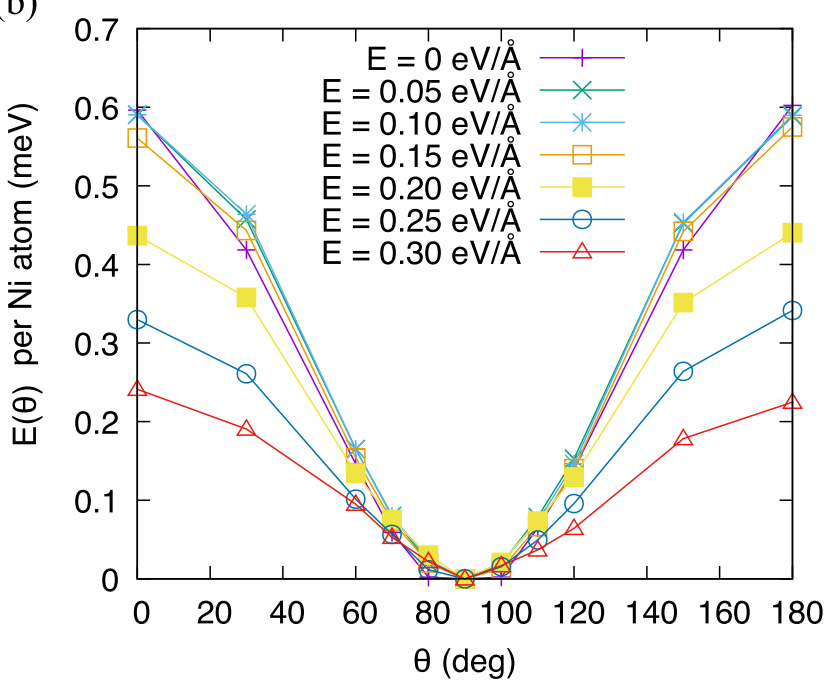

FIG. 5. (a) Magnetic anisotropy energy per Ni atom as a function of external electric fields. (b) Relative energy vs the polar angle of spin of Ni atoms for the DTN slab under different electric fields. For each electric field, the minimal energy is chosen as a reference. The data points are calculated results and the lines are for guiding the eyes.

carry electric dipoles as well and the re-orientation of these molecules does not cost much energy because intermolecular interactions are usually weak. Putting these molecule on a disordered substrate can lead to magnetic chains made up of molecules with random orientation.

It is worth noting that there are, inevitably, some random magnetic fields present in disordered quantum magnets. For example, $\mathrm{Mn}_{12}$-ac crystals have been theorectically predicted [60] and then experimentally confirmed [61] to be a realization of random-field Ising ferromagnetism. The randomness is due to the disorder of the acetic acid of crystallization that induces small but finite distortion of Mn sites, resulting a misalignment of the easy axis [62].

Experimentally probing MBL is a challenging task, especially in natural solid state systems. One of the experimental signatures can be the remanent magnetization after a quench

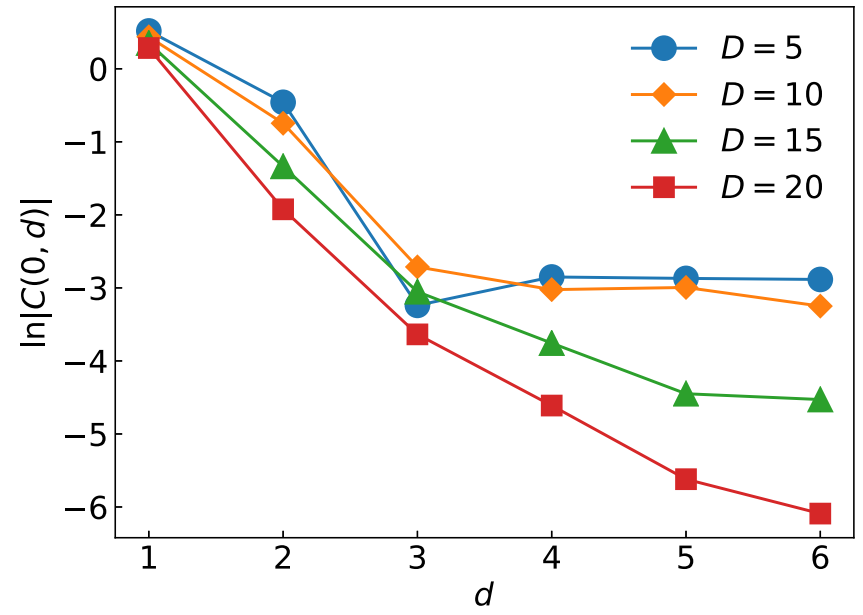

FIG. 6. The spin-spin correlation as a function of the distance $d$ for different $D$. Each data point is an average over 200 samples with size $L=12$. The correlation is independent of $d$ in the thermal phase $(D=5)$ and decays exponentially with $d$ in the localized phase $(D=20)$.

setup. Initially a strong field is needed to fully polarize the spin chain. In the thermal phase, the initial magnetization is completely relaxed, while a finite remanent magnetization is expected in the localized phase [63]. Another probe is the spin-spin correlation function, which can be measured by magnetic neutron scattering, and provides a criterion that distinguishes the thermal and MBL phases [5]. The spin-spin correlation function of two spins at site $i$ and $j$ for eigenstate $|n\rangle$ is given by

$$
C_{n}(i, j)=\left\langle n\left|S_{i}^{z} S_{j}^{z}\right| n\right\rangle-\left\langle n\left|S_{i}^{z}\right| n\right\rangle\left\langle n\left|S_{j}^{z}\right| n\right\rangle .
$$

Figure 6 shows $\ln \left|C_{n}(0, d)\right|$ averaged over eigenstates and samples as a function of the distance $d$, and the behavior of the correlation is different between the thermal and MBL phase. When $D<D^{*}$, i.e., in the thermal phase, the spins are entangled and correlated even if far apart; the correlation between two well-separated sites is independent of their distance, as shown by $D=5$ in Fig. 6 . On the contrary, in the localized phase the correlation between two spins decay exponentially with their distance as can be seen in $D=20$.

The inevitable coupling between electrons and the phonon bath in solids is a well-known obstacle for experimentally observing Anderson localization [64] and also destabilizes MBL [65]. Diagnostics for MBL in the spectral function, including its inhomogenity and the zero frequency gap are present as long as the coupling is smaller than the characteristic energy scales of the system [66]. A recent study proposed that driving the system by light can compensate the effect of phonons [67]. In their work, large site-to-site variations of local temperature was used as a criterion for the MBL phase, and can be measured by various techniques, e.g., thermoreflectance [68].

\section{CONCLUSION AND OUTLOOK}

We have shown that, in absence of magnetic anisotropy $S=1$ has a similar behavior to $S=1 / 2$ chain. MBLT occurs at $W_{h}^{*} \simeq 7.09$, significantly larger than that of $S=1 / 2 . W_{h}^{*}$ decreases with an increasing uniform anisotropy and ap- 
proaches a limit around 4. A large anisotropy reduces $W_{h}^{*}$ and random fields are not needed when $|D / J| \gtrsim 10$. Considering that it is difficult to generate large random local magnetic fields in natural materials but much easier to create disorder with random anisotropy axes, this numerical study paves the way to realizing MBL in natural electronic materials. We discussed the possibility of using organometallic quantum magnets for this purpose, and proposed two candidate compounds NENC and NBYC.

The difficulty of numerically studying chains with higher spins or spin systems beyond 1D greatly limited the size of the system we considered. New numerical and theoretical tools are needed. It was reported recently that Dirac-Frenkel timedependent variational principle as applied to matrix product states is able to study chains up to 100 sites [69]. The presence of phonons is apparently the biggest obstacle for experimen- tally realizing MBL. Further study is needed to address how to compensate the effect of phonons and what experimental signatures can be used in the presence of phonons.

\section{ACKNOWLEDGMENTS}

This work was supported as part of the Center for Molecular Magnetic Quantum Materials ( $\left.\mathrm{M}^{2} \mathrm{QM}\right)$, an Energy Frontier Research Center funded by the US Department of Energy, Office of Science, Basic Energy Sciences under Award DESC0019330. H.-P.C. also acknowledges support from the US Department of Energy (DOE), Office of Basic Energy Sciences (BES), under Contract No. DE-FG02-02ER45995. Computations were done using the utilities of the National Energy Research Scientific Computing Center and University of Florida Research Computing.
[1] D. Basko, I. Aleiner, and B. Altshuler, Ann. Phys. (NY) 321, 1126 (2006).

[2] I. V. Gornyi, A. D. Mirlin, and D. G. Polyakov, Phys. Rev. Lett. 95, 206603 (2005).

[3] J. Z. Imbrie, Phys. Rev. Lett. 117, 027201 (2016).

[4] V. Oganesyan and D. A. Huse, Phys. Rev. B 75, 155111 (2007).

[5] A. Pal and D. A. Huse, Phys. Rev. B 82, 174411 (2010).

[6] J. A. Kjäll, J. H. Bardarson, and F. Pollmann, Phys. Rev. Lett. 113, 107204 (2014).

[7] D. J. Luitz, N. Laflorencie, and F. Alet, Phys. Rev. B 91, 081103(R) (2015).

[8] M. Schreiber, S. S. Hodgman, P. Bordia, H. P. Luschen, M. H. Fischer, R. Vosk, E. Altman, U. Schneider, and I. Bloch, Science 349, 842 (2015).

[9] S. S. Kondov, W. R. McGehee, W. Xu, and B. DeMarco, Phys. Rev. Lett. 114, 083002 (2015).

[10] J.-y. Choi, S. Hild, J. Zeiher, P. Schauss, A. Rubio-Abadal, T. Yefsah, V. Khemani, D. A. Huse, I. Bloch, and C. Gross, Science 352, 1547 (2016).

[11] J. Smith, A. Lee, P. Richerme, B. Neyenhuis, P. W. Hess, P. Hauke, M. Heyl, D. A. Huse, and C. Monroe, Nat. Phys. 12, 907 (2016).

[12] G. Kucsko, S. Choi, J. Choi, P. C. Maurer, H. Zhou, R. Landig, H. Sumiya, S. Onoda, J. Isoya, F. Jelezko, E. Demler, N. Y. Yao, and M. D. Lukin, Phys. Rev. Lett. 121, 023601 (2018).

[13] T. Kohlert, S. Scherg, X. Li, H. P. Lüschen, S. Das Sarma, I. Bloch, and M. Aidelsburger, Phys. Rev. Lett. 122, 170403 (2019).

[14] K. X. Wei, C. Ramanathan, and P. Cappellaro, Phys. Rev. Lett. 120, 070501 (2018)

[15] P. W. Anderson, Phys. Rev. 109, 1492 (1958).

[16] R. Nandkishore and D. A. Huse, Annu. Rev. Condens. Matter Phys. 6, 15 (2015).

[17] D. A. Abanin and Z. Papić, Ann. Phys. (Berl.) 529, 1700169 (2017).

[18] F. Alet and N. Laflorencie, Comptes Rendus Physique 19, 498 (2018).

[19] D. A. Abanin, E. Altman, I. Bloch, and M. Serbyn, Rev. Mod. Phys. 91, 021001 (2019).
[20] J. M. Deutsch, Phys. Rev. A 43, 2046 (1991).

[21] M. Srednicki, Phys. Rev. E 50, 888 (1994).

[22] J. E. Graebner, B. Golding, and L. C. Allen, Phys. Rev. B 34, 5696 (1986).

[23] S. John, Phys. Rev. Lett. 58, 2486 (1987).

[24] D. S. Wiersma, P. Bartolini, A. Lagendijk, and R. Righini, Nature (London) 390, 671 (1997).

[25] V. V. Kruglyak, S. O. Demokritov, and D. Grundler, J. Phys. D 43, 264001 (2010).

[26] M. Serbyn, Z. Papić, and D. A. Abanin, Phys. Rev. Lett. 110, 260601 (2013).

[27] D. A. Huse, R. Nandkishore, V. Oganesyan, A. Pal, and S. L. Sondhi, Phys. Rev. B 88, 014206 (2013).

[28] R. Alben, J. J. Becker, and M. C. Chi, J. Appl. Phys 49, 1653 (1978).

[29] R. Bruinsma and S. N. Coppersmith, Phys. Rev. B 33, 6541 (1986).

[30] R. A. Serota, Phys. Rev. B 37, 9901 (1988).

[31] V. S. Amaral, B. Barbara, J. B. Sousa, and J. Filippi, EPL 22, 139 (1993).

[32] R. Skomski, H. Zeng, M. Zheng, and D. J. Sellmyer, Phys. Rev. B 62, 3900 (2000).

[33] M. Evers, C. A. Müller, and U. Nowak, Phys. Rev. B 92, 014411 (2015).

[34] B. Tang, D. Iyer, and M. Rigol, Phys. Rev. B 91, 161109(R) (2015).

[35] P. Sierant, D. Delande, and J. Zakrzewski, Phys. Rev. A 95, 021601(R) (2017).

[36] Y. Y. Atas, E. Bogomolny, O. Giraud, and G. Roux, Phys. Rev. Lett. 110, 084101 (2013).

[37] F. Pietracaprina, N. Macé, D. J. Luitz, and F. Alet, SciPost Phys. 5, 045 (2018).

[38] M. Serbyn, Z. Papic, and D. A. Abanin, Phys. Rev. X 5, 041047 (2015).

[39] G. Christou, D. Gatteschi, D. N. Hendrickson, and R. Sessoli, MRS Bull. 25, 66 (2000).

[40] C. Coulon, H. Miyasaka, and R. Clérac, in Single-Molecule Magnets and Related Phenomena (Springer, Berlin, Heidelberg, 2006), pp. 163-206.

[41] M. Kurmoo, Chem. Soc. Rev. 38, 1353 (2009). 
[42] R. Yu, L. Yin, N. S. Sullivan, J. S. Xia, C. Huan, A. PaduanFilho, N. F. Oliveira, Jr., S. Haas, A. Steppke, C. F. Miclea, F. Weickert, R. Movshovich, E.-D. Mun, B. L. Scott, V. S. Zapf, and T. Roscilde, Nature (London) 489, 379 (2012).

[43] K. Bernot, L. Bogani, A. Caneschi, D. Gatteschi, and R. Sessoli, J. Am. Chem. Soc. 128, 7947 (2006).

[44] S. Hill, R. S. Edwards, N. Aliaga-Alcalde, and G. Christou, Science 302, 1015 (2003).

[45] A. Ardavan, O. Rival, J. J. L. Morton, S. J. Blundell, A. M. Tyryshkin, G. A. Timco, and R. E. P. Winpenny, Phys. Rev. Lett. 98, 057201 (2007).

[46] C. Schlegel, J. van Slageren, M. Manoli, E. K. Brechin, and M. Dressel, Phys. Rev. Lett. 101, 147203 (2008).

[47] S. Takahashi, I. S. Tupitsyn, J. van Tol, C. C. Beedle, D. N. Hendrickson, and P. C. E. Stamp, Nature (London) 476, 76 (2011).

[48] M. Shiddiq, D. Komijani, Y. Duan, A. Gaita-Ariño, E. Coronado, and S. Hill, Nature (London) 531, 348 (2016).

[49] J. M. Zadrozny, J. Niklas, O. G. Poluektov, and D. E. Freedman, ACS Cent. Sci. 1, 488 (2015).

[50] Y.-Q. Zheng and H.-Z. Xie, J. Solid State Chem. 177, 1352 (2004).

[51] A. D. Katsenis, R. Inglis, A. Prescimone, E. K. Brechin, and G. S. Papaefstathiou, Cryst. Eng. Comm. 14, 1216 (2012).

[52] M. Abel, S. Clair, O. Ourdjini, M. Mossoyan, and L. Porte, J. Am. Chem. Soc. 133, 1203 (2011).

[53] M.-L. Tong, S. Kitagawa, H.-C. Chang, and M. Ohba, Chem. Commun. 4, 418 (2004).

[54] W. J. L. Buyers, R. M. Morra, R. L. Armstrong, M. J. Hogan, P. Gerlach, and K. Hirakawa, Phys. Rev. Lett. 56, 371 (1986).
[55] M. T. Batchelor, X.-W. Guan, and N. Oelkers, Phys. Rev. B 70, 184408 (2004).

[56] M. Orendáč, A. Orendáčová, J. Černák, A. Feher, P. J. C. Signore, M. W. Meisel, S. Merah, and M. Verdaguer, Phys. Rev. B 52, 3435 (1995).

[57] A. Feher, M. Orendáč, A. Orendáčová, and E. Čižmár, Low Temp. Phys. 28, 551 (2002).

[58] C. van Wüllen, J. Chem. Phys. 130, 194109 (2009).

[59] V. S. Zapf, P. Sengupta, C. D. Batista, F. Nasreen, F. WolffFabris, and A. Paduan-Filho, Phys. Rev. B 83, 140405(R) (2011).

[60] A. J. Millis, A. D. Kent, M. P. Sarachik, and Y. Yeshurun, Phys. Rev. B 81, 024423 (2010).

[61] B. Wen, P. Subedi, L. Bo, Y. Yeshurun, M. P. Sarachik, A. D. Kent, A. J. Millis, C. Lampropoulos, and G. Christou, Phys. Rev. B 82, 014406 (2010).

[62] A. Cornia, R. Sessoli, L. Sorace, D. Gatteschi, A. L. Barra, and C. Daiguebonne, Phys. Rev. Lett. 89, 257201 (2002).

[63] V. Ros and M. Müller, Phys. Rev. Lett. 118, 237202 (2017).

[64] P. A. Lee and T. V. Ramakrishnan, Rev. Mod. Phys. 57, 287 (1985).

[65] R. Nandkishore and S. Gopalakrishnan, Ann. Phys. (Berl.) 529, 1600181 (2017).

[66] S. Johri, R. Nandkishore, and R. N. Bhatt, Phys. Rev. Lett. 114, 117401 (2015).

[67] Z. Lenarčič, E. Altman, and A. Rosch, Phys. Rev. Lett. 121, 267603 (2018).

[68] D. G. Cahill, W. K. Ford, K. E. Goodson, G. D. Mahan, A. Majumdar, H. J. Maris, R. Merlin, and S. R. Phillpot, J. Appl. Phys. 93, 793 (2003).

[69] E. V. H. Doggen, F. Schindler, K. S. Tikhonov, A. D. Mirlin, T. Neupert, D. G. Polyakov, and I. V. Gornyi, Phys. Rev. B 98, 174202 (2018). 